\title{
A 3D Polygonal Line Chains Matching Method for Face Recognition
}

\author{
Xun Yu, Yongsheng Gao \\ School of Engineering \\ Griffith University \\ Nathan, QLD 4111, Australia \\ xun.yu@griffithuni.edu.au,yongsheng.gao@griffith.edu.au
}

\author{
Jun Zhou \\ School of Information and Communication Technology \\ Griffith University \\ Nathan, QLD 4111, Australia \\ Jun.zhou@griffith.edu.au
}

\begin{abstract}
In this paper, a novel 3D polygonal line chains matching method is proposed. Different from traditional method that use points and meshes to represent and match 3D shapes, our method represents 3D surfaces using 3D polygonal line chains generated from ridge and valley curves. Then a 3D polygonal line segment Hausdorff distance measure is developed to compute the similarity between two 3D surfaces. This representation, along with the distance metric, can effectively harness structural and spatial information on a 3D surface. The added information can provide more and better discrimination power for object recognition. It strengthens and improves the matching process of similar 3D objects such as 3D faces. Experiments on FRGC v2 database leads to a rank one recognition rate of $96.1 \%$.
\end{abstract}

Keywords-3D polygonal line chains; $3 D$ polygonal line segment Hausdorff distance; 3D Face Recognition.

\section{INTRODUCTION}

Automatic face recognition has apparent advantages over other biometric technologies due to its natural, nonintrusive, and high throughput properties. Over the past three decades, most efforts in this area have been devoted to face recognition from 2D images [1]. Although 2D face recognitions can achieve good performance under controlled environments, i.e. when gallery and probe images are in the similar condition, they still encounter various problems in uncontrolled conditions, such as variations in illumination, pose, expression and age, because a $2 \mathrm{D}$ image is a projection of a $3 \mathrm{D}$ face with one dimension lost [2].

Due to the rapid development and dropping cost of $3 \mathrm{D}$ sensors, 3D face recognition methods are now receiving increasingly attention from the research community. Compared to conventional $2 \mathrm{D}$ face recognition, 3D face recognition is expected to be less sensitive to illumination and pose variations. This is due to the face structure that can be recovered from $3 \mathrm{D}$ data, which is related to the internal face anatomy instead of external appearance and environment [3]. In addition, the geometric information in 3D data provides more discriminatory features for face recognition.

In this paper, a novel 3D polygonal line chains (PLC) matching method is proposed for face recognition. This approach reduces storage requirement by grouping ridge and valley curves into PLCs. Moreover, we offer a 3D polygonal line segment Hausdorff distance (3D PLHD) measure to match 3D PLCs of human faces. Compared to conventional application of Hausdorff distance, 3D PLHD has better discriminative power because it makes use of the additional attributes of line orientation and line-point association.

The rest of this paper is organized as follows. Section 2 discusses related work in the area of $3 \mathrm{D}$ face recognition. Section 3 presents the proposed 3D polygonal line chains. Section 4 presents our 3D polygonal line chains matching method. Encouraging experimental results on a public database are reported in Section 5. Finally, conclusions are drawn in Section 6.

\section{RELATED WORK}

A comprehensive review of $3 \mathrm{D}$ face recognition methods can be found in [4]. Here, we will not perform a thorough review of research in $3 \mathrm{D}$ face recognition, but instead mention those findings more relevant to our paper.

Mahoor and Mohamed [5] encoded the range data of 3D face into a ridge image, which showed the locations of ridge lines around the important facial regions on the face (i.e eyes, nose, and mouth). A least trimmed square Hausdorff distance [6] was utilized to match the $3 \mathrm{D}$ points lying on ridge image of a given probe to the created ridge images of the subjects in the gallery. In their work, only about $14 \%$ of the total number of points on the range data were used, but achieved $93.5 \%$ accuracy on GavabDB database which contains images from 61 subjects. However, without considering the inherent local structural characteristics inside such images, the Hausdorff distance uses only the spatial information of ridge image. The approach described in this paper harnesses the structural and spatial information of ridge image. In addition, compared to [5], our approach further reduces the storage demand .

Kim et al. [7] proposed a face recognition algorithm using combined depth information on the edge obtained by stereo camera and the conventional Line Edge Map (LEM) [8]. To match two faces, the LEM approach was first implemented to extract line segments from a face edge map. Then, the corresponding depth information was added to the end points of each line segment and formed a 3D LEM. A modified line segment Hausdorff distance [9] was then proposed to measure the similarity of two 3D LEMs. Experimental results showed that the recognition rate of their approach increased $1.3 \% \sim$ $7.8 \%$ over that of the conventional 2D LEM approach under 


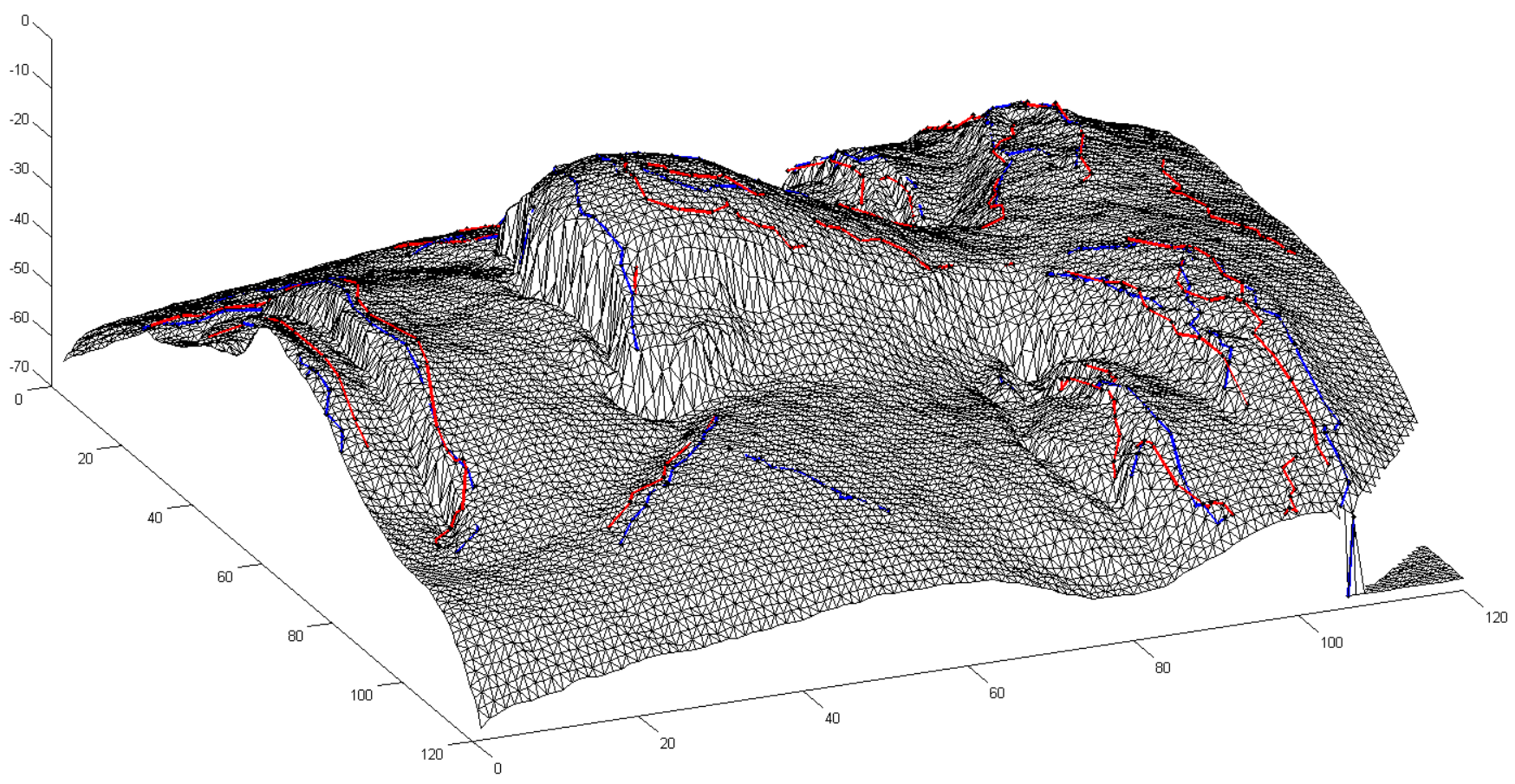

Figure 1. Describing 3D shapes using 3D polygonal line Chains.

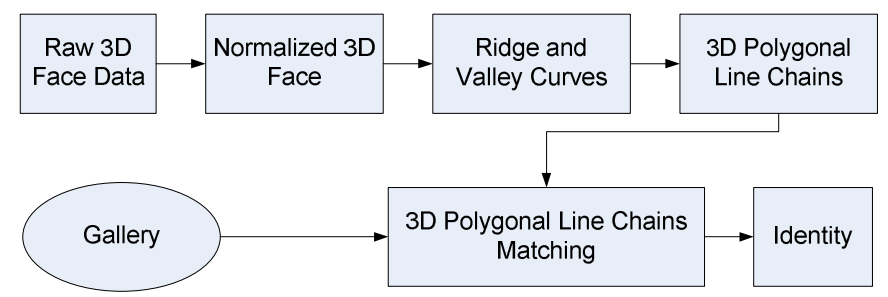

Figure 2. A flowchart of the proposed approach.

conditions like face expression changes and facial occlusions. This shows that the increase in performance can be achieved by using 3D data. However, the approach in [7] is not a complete 3D face recognition method and still needs using 2D images. Moreover, simply adding depth information into 2D end points of line segment does not consider the inherent information of 3D objects. On the contrary, the proposed approach directly extracts feature lines from $3 \mathrm{D}$ face and utilizes additional 3D geometry information in the matching process.

\section{3D Polygonal Line Chains}

Currently, points and meshes are widely used as the features to describe and match 3D shapes [10]. Relevant methods employ the spatial information of these features for similarity measurement. The disadvantage of such approaches are prominent, i.e., they lack of local structural discriminative capability.
In this paper, we propose a new 3D shape representation, the 3D polygonal line chains (PLCs), which combines the structural information with spatial information. It groups the ridge and valley curves into two distinctive sets of 3D line chains to enable the effective description of local structural characteristics of salient curves on the 3D surface. As demonstrated in [11], ridge and valley curves on a surface along which the surface bends sharply are geometrically and perceptually salient surface features. Therefore, they can be used for shape coding and recognition. After using the algorithm in [11], the 3D face are featured into a ridge line set and a valley line set, according to their curvature directions on the surface. Each line set contains a number of curves that are composed of short ridge (or valley) line segments. The Douglas-Peucker algorithm [12] is then applied to generate the 3D PLCs of the two line sets, which further reduces the number of points on the curves.

Figure 1 shows the 3D PLCs of a 3D face from FRGC v2 database. Compared to ridge and valley curves, 3D PLCs can be regarded as a higher level face coding method. It contains local structural characteristics by grouping connected ridge (or valley) line segments into a polygonal line. The orientation of such polygonal line, which is a kind of local structural representation, is more robust to the noise caused by 3D scanner than that of ridge and valley line segments. The polygonal line segment is the basic element of 3D PLC. A method to calculate the similarity between two 3D PLCs will be described in the next section. The whole algorithm flow is illustrated in Figure 2. 


\section{3D Polygonal Line Chains Matching}

\section{A. 3D Hausdorff Distance}

The Hausdorff distance is a measure for shape comparison in computer vision. It was originally used for point sets in the 2D plane [13] and then also applied to points in 3D space [14]. Unlike most shape matching methods, Hausdorff distance can be calculated without one-to-one correspondence of points. The undirected Hausdorff distance between two point sets $G$ and $\mathrm{P}$ is presented in equation (1).

$$
H(G, P)=\max (h(G, P), h(P, G)),
$$

where $h(G, P)$ denotes the directed Hausdorff distance

$$
h(G, P)=\max _{g \in G} \min _{p \in P}\|g-p\|,
$$

and $\|g-p\|$ is some underlying norm. In most cases, the Euclidean distance is used here.

In [15], the modified Hausdorff distance (MHD) was proved experimentally to be the most effective one among 24 different forms of Hausdorff distance. Here we extend the MHD to 3D space. Same as 2D MHD, the directed 3D MHD is defined as

$$
h(G, P)=\frac{1}{N_{g}} \sum_{g \in G} \min _{p \in P}\|g-p\|
$$

where $\mathrm{G}$ and $\mathrm{P}$ are two $3 \mathrm{D}$ point sets (i.e. range data) and $N_{g}$ is the number of points in G. The definition of undirected $3 \mathrm{D}$ MHD is the same as equation (1).

Such 3D MHD method is more robust to outlier points compared to generalized 3D Haudsorff distance. However, it still lacks the capability in using structural representation of $3 \mathrm{D}$ shape (i.e. the orientation information). Nonetheless, the proposed 3D PLHD can harness the spatial information and structural information of $3 \mathrm{D}$ shape and can be expected to increase the recognition performance.

\section{B. Proposed 3D Polygonal Line Segment Hausdorff Distance}

We start from definition of two 3D polygonal line chains $G=G^{r} \cup G^{v}=\left\{g_{1}^{r}, g_{2}^{r}, \cdots, g_{p}^{r}, g_{1}^{v}, g_{2}^{v}, \cdots, g_{q}^{v}\right\}$ and $P=P^{r} \cup P^{v}=\left\{p_{1}^{r}, p_{2}^{r}, \cdots, p_{s}^{r}, p_{1}^{v}, p_{2}^{v}, \cdots, p_{t}^{v}\right\}$ that represent a gallery $3 \mathrm{D}$ shape and a probe $3 \mathrm{D}$ shape respectively, where superscripts $r$ and $v$ stand for ridge and valley. $G$ consists of two subset $G^{r}$ and $G^{v}$ that correspond to 3D line chains along ridge and valley curves. 3D PLHD is built on $d\left(g_{i}^{k}, p_{j}^{k}\right)(\mathrm{k}$ represents $\mathrm{r}$ or $\mathrm{v}$ ) that calculates the distance between two 3D polygonal line segments belong to $G^{k}$ and $\mathrm{P}^{k} \cdot d\left(g_{i}^{k}, p_{j}^{k}\right)$ consists of three aspects of difference between two 3D polygonal line segments: perpendicular distance $\left(d_{\perp}\right)$, parallel distance $\left(d_{\|}\right)$and orientation distance $\left(d_{\theta}\right)$. They are defined as:

$$
\begin{gathered}
d_{\perp}\left(g_{i}^{k}, p_{j}^{k}\right)=l_{\perp}, \\
d_{\|}\left(g_{i}^{k}, p_{j}^{k}\right)=\min \left(l_{\| 1}, l_{\| 2}\right), \\
d_{\theta}\left(g_{i}^{k}, p_{j}^{k}\right)=f\left(\theta\left(g_{i}^{k}, p_{j}^{k}\right)\right),
\end{gathered}
$$

where

$$
\theta\left(g_{i}^{k}, p_{j}^{k}\right)=\cos ^{-1}\left(\left|\hat{u}_{g_{i}^{k}} \cdot \hat{u}_{p_{j}^{k}}\right|\right)
$$

In this equation, $\hat{u}_{g_{i}^{k}}$ and $\hat{u}_{p_{j}^{k}}$ are the unit direction vector of $g_{i}^{k}$ and $p_{j}^{k}$. In equation (6), $f(\theta)=\tan \theta$ ( $\theta$ is capped by $89^{\circ}$ to avoid calculating $\tan 90^{\circ}$ ) is a non-linear penalty function to penalize large angle deviation but ignore small variation.

As shown in Figure 3, parallel distance is the minimum displacement to align either the left end points or the right points of the lines. Perpendicular distance is the vertical distance between the lines. If $g_{i}^{k}$ and $p_{j}^{k}$ are not parallel, the shorter line is rotated along its midpoint to be parallel with the longer line before calculating the parallel distance and the perpendicular distance, since less distortion to the original line pairs would be caused. An example of the 3D polyline segment rotation is illustrated in Figure 4. To demonstrate the rotation process in $3 \mathrm{D}$ space, the projection of each polyline segment is also shown here.

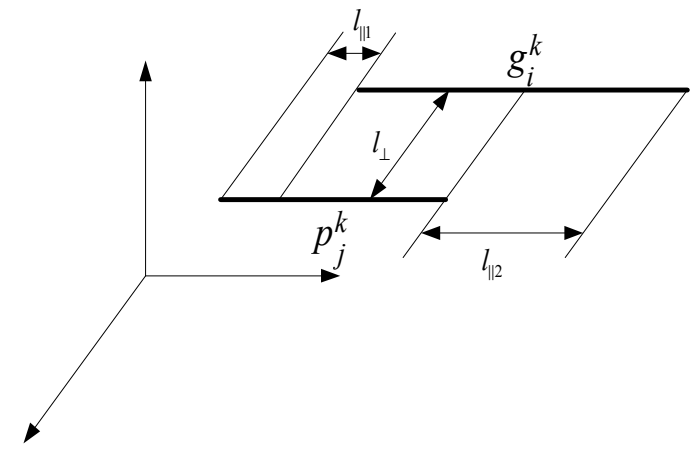

Figure 3. An illustration of perpendicular and parallel distances.

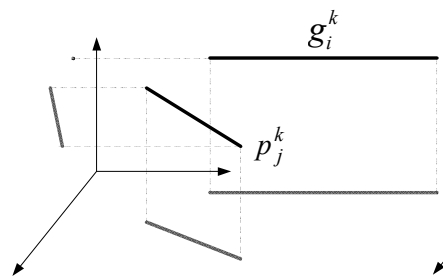

(a)

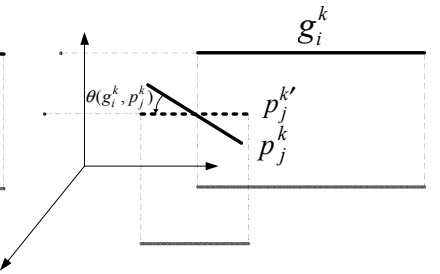

(b)
Figure 4. Rotation of shorter 3D polygonal line segment. (a) The original line pairs. (b) The shorter line rotated. The dashed line is the one after rotation. 
Finally, the distance between two line segments can be calculated as the Euclidean distance of all three distances mentioned above:

$$
d\left(g_{i}^{k}, p_{j}^{k}\right)=\sqrt{d_{\perp}\left(g_{i}^{k}, p_{j}^{k}\right)^{2}+d_{\|}\left(g_{i}^{k}, p_{j}^{k}\right)^{2}+\left(W_{o} \cdot d_{\theta}\left(g_{i}^{k}, p_{j}^{k}\right)\right)^{2}}
$$

where $W_{o}$ is the weight for the orientation distance to be determined by a training process.

Based on the distance in equation (8), the 3D PLHD is defined as

$$
\begin{aligned}
& H_{3 D P L H D}(G, P)=H_{3 D P L H D}\left(G^{r}, P^{r}\right)+H_{3 D P L H D}\left(G^{v}, P^{v}\right) \\
& =\max \left(h\left(G^{r}, P^{r}\right), h\left(P^{r}, G^{r}\right)\right)+\max \left(h\left(G^{v}, P^{v}\right), h\left(P^{v}, G^{v}\right)\right)
\end{aligned}
$$

where

$$
h\left(G^{r}, P^{r}\right)=\frac{1}{\sum_{g_{i}^{r} \in G^{r}} l_{g_{i}^{r}}} \sum_{g_{i}^{r} \in G^{r}} l_{g_{i}^{r}} \cdot \min _{p_{j}^{r} \in P^{r}} d\left(g_{i}^{r}, p_{j}^{r}\right)
$$

In this equation, $l_{g_{i}^{r}}$ is the length of $3 \mathrm{D}$ polygonal line segment $g_{i}^{r}$. The distance contribution from each 3D polygonal line segment is weighted by its length. In equation (9), the 3D PLHD between gallery and probe is merged from 3D PLHD between ridge subset and valley subset. This fusion process can further improve the discriminative power.

\section{EXPERIMENTAL RESULTS}

In our experiments, we used the FRGC v2 3D database [16] which is one of the largest available 3D facial images database. This database contains 466 persons collected in 4007 sessions (from 1 to 22 sessions per person). 388 persons have more than one session with neutral expressions to be used in our experiments. The $3 \mathrm{D}$ images were acquired with a Minolta Vivid 900/910 series laser scanner under controlled illumination conditions. In the FRGC v2, 3D images consist of both range and texture channels. Here, we only use the range data. In the following experiments, the performance of the proposed method is compared with 3D MHD method without $3 \mathrm{D}$ polyline feature and the method proposed by Mahoor and Mohamed [5].

\section{A. Determination of Parameter $W_{o}$}

In this section, we investigate the effect of parameter $W_{o}$ in equation (8) to determine a value of $W_{o}$ which provides the 3D PLHD with a better recognition performance. To do so, a 100person training dataset from the FRGC v2 was created. In this training set, for each person, the neutral 3D face in the first session was selected as the gallery image, and the neutral 3D face in the second session was used as the probe image. The recognition rate is plotted against the values of $W_{o}$ in Figure 5 . It is observed that $3 \mathrm{D}$ PLHD with a low value of $W_{o}$ performed badly. The performance increased quickly and reached the optimal value when $W_{o}$ range from 0 to 25 . In the rest of experiments in this study, $W_{o}$ is set as 20.

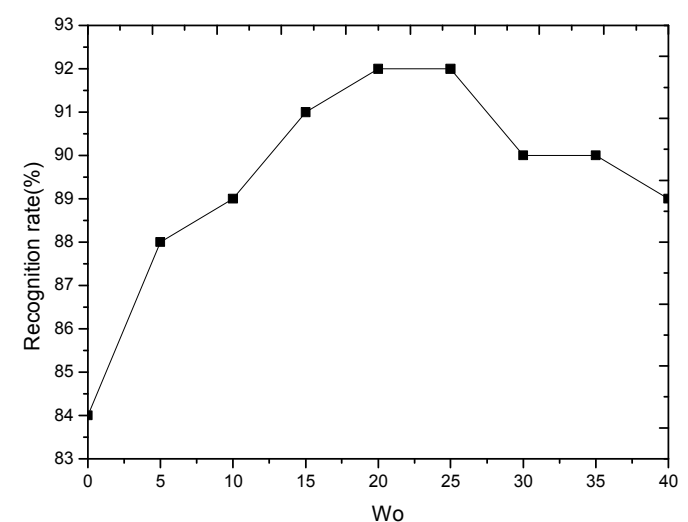

Figure 5. Recognition rate versus the value of $W_{o}$.

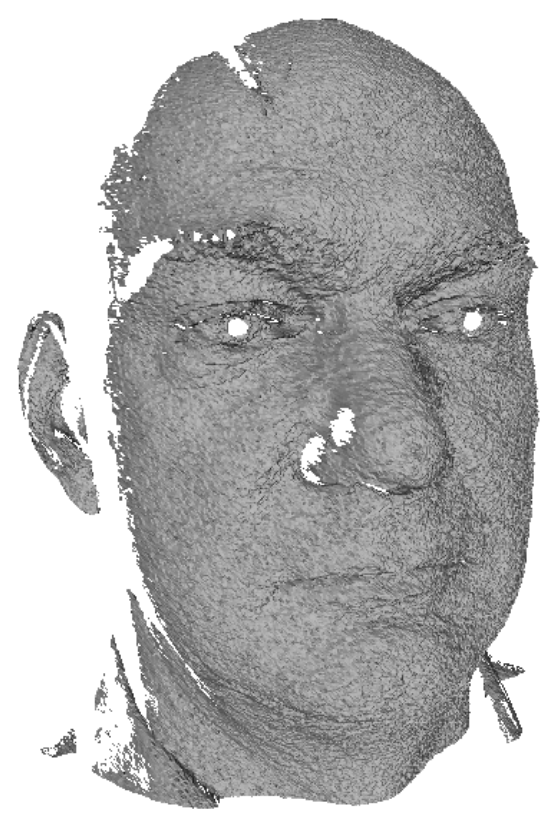

Figure 6. An example 3D face in the FRGC database.

\section{B. Face Recognition}

In our experiments, 388 persons with 2 neutral 3D faces per person from the FRGC v2 were used to test the effectiveness of the proposed approach. For each person, one neutral 3D face was used as a model while the other was used as a test.

Figure 6 shows an example of the raw 3D face extracted from the dataset. The raw data contains a number of imperfections, such as holes, spikes, and includes some nonfacial regions, such as neck, ears. Prior to applying our algorithm, the face images were extracted, normalized, aligned and cropped in the same manner as in [17]. Spikes in the range data were removed and holes were filled. Figure 7 illustrates samples of normalized and copped faces in the FRGC v2 database and their corresponding ridge and valley data. 

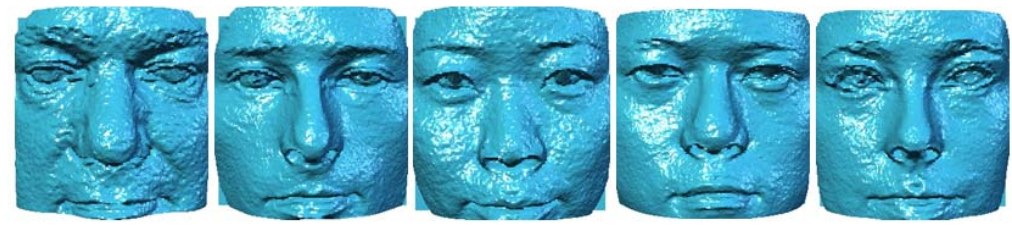

(a)
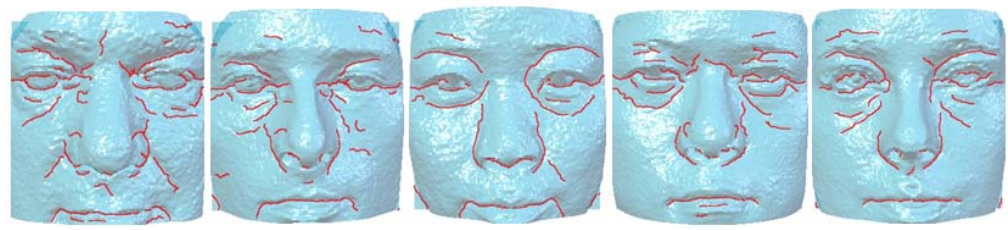

(b)
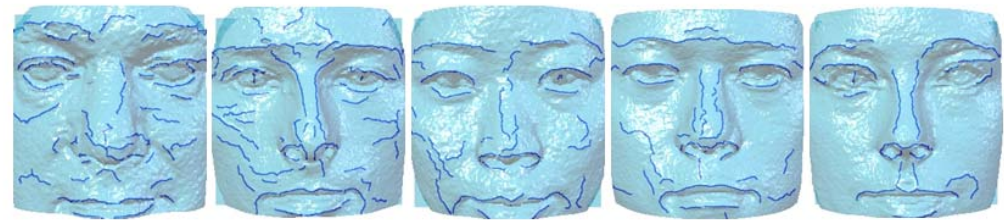

(c)

Figure 7. Samples of the faces used in our tests. (a) Normalized faces. (b) Ridge data. (c) Valley data.

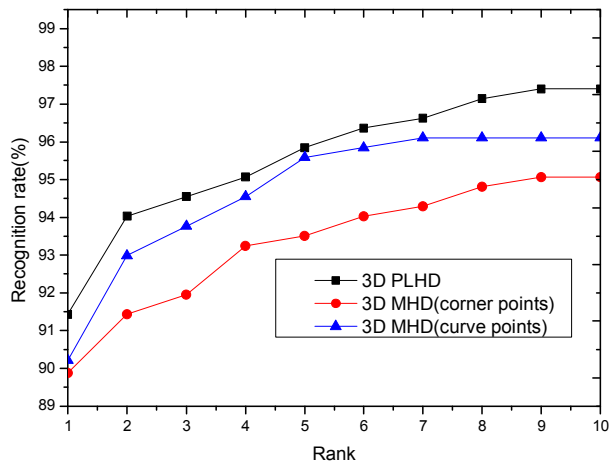

Figure 8. CMC curves of the proposed method against benchmark methods using ridge data.

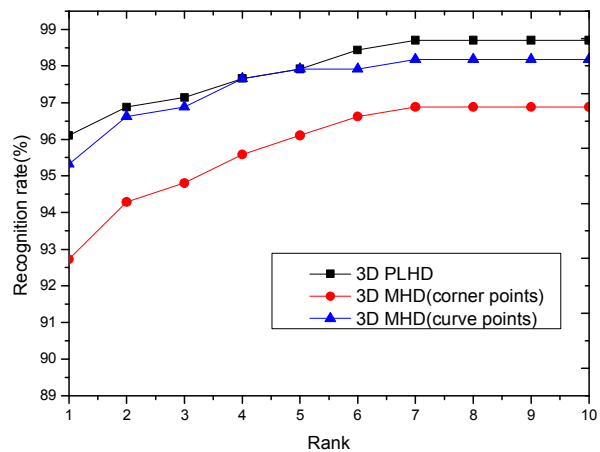

Figure 9. CMC curves of the proposed method against benchmark methods using valley data.

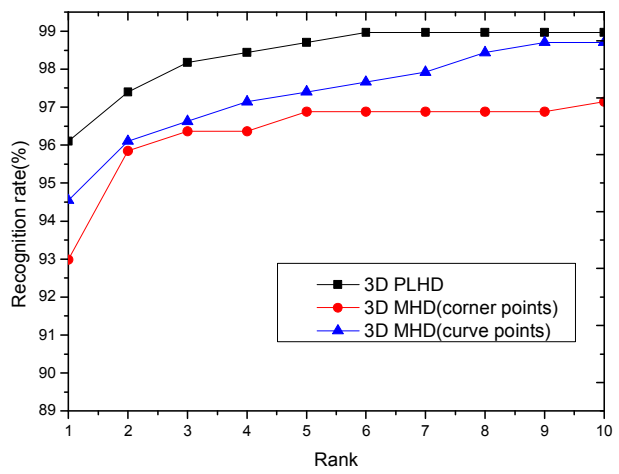

Figure 10. CMC curves of the proposed method against benchmark methods after fusion process.

The performance is measured in terms of the Cumulative Match Characteristics (CMC) [18] and the rank-1 recognition rate. We show the CMC of the proposed method together with 3D MHD methods that based on points lying on ridge and valley curves (curve points) and points lying on 3D PLCs (corner points). In order to demonstrate the performance improvement resulted from the fusion process, we first used data based on ridge and valley curves respectively, as illustrated in Figure 8 and Figure 9. The eventual fusion result is shown in Figure 10. Note that the recognition rate of the proposed method is always higher than the benchmark methods. This demonstrates that the added structural information makes 3D PLHD a more discriminative measure than 3D MHD. 
The rank-1 recognition rates of the methods given in Figure 9 above are tabulated in Table 1 together with the reported results of the Mohammad et al. [5]. It is encouraging to find that 3D PLHD achieved higher score than all other 3 methods.

In addition, compared with 3D MHD (curve points), 3D PLHD requires only $18.9 \%$ storage space but increases the recognition rate from $94.55 \%$ to $96.10 \%$. When the same storage space is used, a higher score increase from $92.99 \%$ of 3D MHD (corner points) to $96.10 \%$ of 3D PLHD is achieved.

Table 1. Recognition accuracies on 3D face scans

\begin{tabular}{|c|c|}
\hline Method & Recognition Result \\
\hline 3D PLHD & $96.10 \%$ \\
\hline 3D MHD(corner points) & $92.99 \%$ \\
\hline 3D MHD(curve points) & $94.55 \%$ \\
\hline Mahoor and Mohamed [5] $*$ & $93.50 \%$ \\
\hline
\end{tabular}

* The result was from the published paper on a 61 person database, while others were obtained on a larger FRGC dataset with 388 persons.

\section{CONCLUSIONS}

This paper presents a novel 3D shape matching method (3D PLHD) based on 3D PLCs representation, which employs both spatial and structural information of ridge and valley curves on 3D surface. Because 3D polygonal line segments other than extreme points (points lying on ridge and valley curves) are used as basic computation unit, the proposed method has achieved better performance than that of conventional pointbased Hausdorff distance method.

The proposed algorithm has been evaluated on FRGC v2 database and compared with 3D MHD and the approach presented by [5]. It is very encouraging to find that the 3D PLHD method has consistently performed superior to these two benchmarks in terms of higher recognition rate and less storage space demand. This research reveals that 3D PLCs provides a new solution for the 3D face recognition, which, may also find its application in general 3D object representation and recognition.

\section{REFERENCES}

[1] W. Zhao, R. Chellappa, P. J. Phillips, and A. Rosenfeld, "Face recognition: A literature survey," ACM Computing Surveys, vol. 35, pp. 399-459, 2003.

[2] G. Medioni and R. Waupotitsch, "Face modeling and recognition in 3$\mathrm{D}, "$ in Proceedings of IEEE International Workshop on Analysis and Modeling of Face and Gestures, pp. 232-233, 2003.

[3] X. G. Lu, A. K. Jain, and D. Colbry, "Matching 2.5D face scans to 3D models," IEEE Transactions on Pattern Analysis and Machine Intelligence, vol. 28, pp. 31-43, 2006.
[4] K. W. Bowyer, K. Chang, and P. Flynn, "A survey of approaches and challenges in $3 \mathrm{D}$ and multi-modal $3 \mathrm{D}+2 \mathrm{D}$ face recognition," Computer Vision and Image Understanding, vol. 101, pp. 1-15, 2006.

[5] M. H. Mahoor and M. Abdel-Mottaleb, "3D face recognition based on $3 \mathrm{D}$ ridge lines in range data," in Proceedings of IEEE International Conference on Image Processing, pp. 137-140, 2007.

[6] D. G. Sim, O. K. Kwon, and R. H. Park, "Object matching algorithms using robust Hausdorff distance measures," IEEE Transactions on Image Processing, vol. 8, pp. 425-429, 1999.

[7] H. M. Kim, H. C. Shin, and S. D. Kim, "Face recognition using 3D Line Edge Map robust to expression changes and occlusions," in Proceedings of the 9th International Conference on Advanced Communication Technology, pp. 357-362, 2007.

[8] Y. S. Gao and M. K. H. Leung, "Face recognition using line edge map," IEEE Transactions on Pattern Analysis and Machine Intelligence, vol. 24, pp. 764-779, 2002.

[9] Y. S. Gao and M. K. H. Leung, "Line segment Hausdorff distance on face matching," Pattern Recognition, vol. 35, pp. 361-371, 2002.

[10] G. K. L. Tam, et al., "Registration of 3D Point Clouds and Meshes: A Survey from Rigid to Nonrigid," IEEE Transactions on Visualization and Computer Graphics, vol. 19, pp. 1199-1217, 2013.

[11] Y. Ohtake, A. Belyaev, and H. P. Seidel, "Ridge-valley lines on meshes via implicit surface fitting," ACM Transactions on Graphics, vol. 23, pp. 609-612, 2004.

[12] D. H. Douglas and T. K. Peucker, "Algorithms for the reduction of the number of points required to represent a digitized line or its caricature," Cartographica: The International Journal for Geographic Information and Geovisualization, vol. 10, pp. 112-122, 1973.

[13] D. P. Huttenlocher, G. A. Klanderman, and W. J. Rucklidge, "Comparing Images Using the Hausdorff Distance," IEEE Transactions on Pattern Analysis and Machine Intelligence, vol. 15, pp. 850-863, 1993.

[14] B. Achermann and H. Bunke, "Classifying range images of human faces with Hausdorff distance," in Proceedings of 15th International Conference on Pattern Recognition, pp. 809-813, 2000.

[15] M. P. Dubuisson and A. K. Jain, "A Modified Hausdorff Distance for Object Matching," in Proceedings of the 12th Iapr International Conference on Pattern Recognition - Conference A: Computer Vision \& Image Processing, pp. 566-568, 1994.

[16] P. J. Phillips, et al., "Overview of the face recognition grand challenge," in Proceedings of IEEE Computer Society Conference on Computer Vision and Pattern Recognition, pp. 947-954, 2005.

[17] A. S. Mian, M. Bennamoun, and R. Owens, "An efficient multimodal 2D-3D hybrid approach to automatic face recognition," IEEE Transactions on Pattern Analysis and Machine Intelligence, vol. 29, pp. 1927-1943, 2007.

[18] S. A. Rizvi, P. J. Phillips, and H. Moon, "The FERET verification testing protocol for face recognition algorithms," in Proceedings of the 3rd International Conference on Automatic Face and Gesture Recognition, pp. 48-53, 1998. 